ein wenig Aufmerksamkeit gelesen hätte, würde er jedenfalls trotz des schlechten Textzustandes bemerkt haben, daß Valentinian sich darin gerade über die Ketzerei und die Verfolgungen des Valens beklagt.

Durch die vorstehenden Betrachtungen scheint mir die Annahme beseitigt zu sein, daß die Handschriften Theodorets nach den Parallelautoren oder nach den Quellen corrigiert wären. Ihre Schreiber haben an ein Verfahren, das uns heute so selbstrerständlich scheint, gar nicht gedacht. Freilich werde ich mich wohl hüten zu behaupten, daß das Studium der Überlieferung anderer Autoren immer zu einem ähnlichen Schluß führen würde. De Boor ist zwar für Theophanes (Theophanis Chronographia II 436 ff) gleichfalls zu diesem Schluß gekommen; aber ich sehe z. B., daß Ed. Schwartz (Eusebius Kirchengeschichte III, S. CLXIV ff) für die Auszüge des Josephus festgestellt hat, daß manche Handschriften des Eusebius nach der Quelle corrigiert sind (s. auch de Boor, Georgii Monachi Chronicon LVIf). Es ist übrigens begreiflich, daß an eine solche Nachvergleichung leichter bei wohlbekannten und klar bezeichneten Autoren gedacht wird als bei Urkunden. Ebenso müssen die Citate aus der Bibel besonders beurteilt werden, und das Verfahren der Chronisten, die sich fortwährend der Reihe nach ergänzen, indem sie sich auf dieselben Quellen beziehen, stellt noch einen weitern, wieder ganz verschiedenen Arbeitsmodus dar.

\title{
XI. Die Abschreiber
}

Das Verzeichnis der Abschreiber, das sich S. $366 \mathrm{f}$ dieser Ausgabe findet, beabsichtigt keineswegs, alle Autoren aufzuzählen, die aus der Kirchengeschichte Stellen entlehnt haben. Ich habe mir besonders angelegen sein lassen, die Autoren heranzuziehen, für welche zuverlässige kritische Ausgaben vorliegen, z. B. Georgius Monachus und Theophanes von de Boor sowie das Synaxarium ecclesiae Constantinopolitanae von Delehaye.

Unter den Anleihen aus Theodoret beanspruchen einen Platz für sich die Excerpte aus der Tripartita des Theodorus Lector, z. B. in Theophanes, Julius Polydeukes, den Anecdota Paris. von Cramer Bd. II, S. $87 \mathrm{ff}$, dem Baroccianus 142, Bl. $216 \mathrm{ff}$ (ich habe für diese Excerpta Barocciana die Abschrift de Boors zu Rate ziehen können) und anderwärts. Im allgemeinen hat die Durchforschung dieser zahlreichen Bruchstücke kaum neues Licht über den Zustand des Textes gebracht, und ich habe in Verweisungen zurückhaltend sein können. Die Frage der Epitome (s. darüber Bidez, La tradition manuscrite de Sozomène, S. $44 \mathrm{f}$ und $77 \mathrm{ff}$ ) würde eine besondere Untersuchung 
erfordern, die auf sich zu nehmen der Herausgeber des Theodoret aber weder den Beruf noch das Bedürfnis fühlt.

Die Chronik des Michael Syrus (Buch 7 u. 8) hat uns einen Abriß von einem sehr großen Teil von Theodorets Werk erhalten. Aber diese summarische Übersicht kann nicht die Dienste leisten, die man sonst von so getreuen Abklatschen erwartet, wie es die syrischen Übersetzungen sind, und die Fälle, wo ihr Zeugnis mir anch nur ein wenig Nutzen gebracht hat, sind ziemlich selten. Dasselbe gilt in noch höherem Maß von den syrischen Chroniken, die Chabot mit verschiedenen Mitarbeitern ins Lateinische übersetzt hat: Corpus scriptorum Christianorum orientalium. Scriptores Syri. Versio. Series tertia. Tomus IV. 1903.

Gelasius. Etwas ausführlicher glaube ich von dem Wert handeln zu sollen, der den bei Gelasius Cyzicenus sich findenden Anleihen aus Theodoret (Buch I u. II) beizumessen ist. G. Loeschcke hat eine erste vortreffliche Arbeit über Gelasius (Rheinisches Museum 59.60. 61) geliefert und wird uns hoffentlich bald mit einer kritischen Ausgabe beglücken, welche über diese Persönlichkeit Licht verbreiten kann. Da ich Gelasius nur in seinen Beziehungen gerade zu Theodoret studiert habe, will ich bloß sagen, daß er mir durch seinen Hang zur Lüge und Interpolation eine ganz besondere Stellung einzunehmen scheint. Geradezu gefährlich macht ihn der Umstand, daß er sich vornehmlich darin gefällt, seine Fälschungen in die Urkunden einzuführen, die doch unantastbar sein sollten.

Ich habe in den Actenstücken gegenüber seinem Text, wo niemand sonst seine Version bestätigt, das größte Mißtrauen, selbst wenn der Schein zu seinen Gunsten ist.

Gelasius gibt zum Beispiel III 18 den Brief Constantins an das Concil von Tyrus wieder, der sich gleichfalls bei Athanasius Apologia contra A rian os 86, beiSocratesI 34 und bei SozomenusII 28 findet. G. Loesch cke (Rhein. Mus.61, S.34ff) glaubt, Gelasius gebe (im Anschluß an Johannes?) eine vollständigere Version von Constantins Brief als die drei andern. Nach seiner Meinung schreiben Socrates und Sozomenus von Athanasius ab. Das ist möglich, aber nicht sicher, da dies Actenstück auch in einer andern Sammlung Aufnahme gefunden hatte.

Der gegenwärtige Text des Socrates, der oft den Anschein hat, ziemlich abweichend zu sein, kann schon mit Hülfe der Übersetzung Cassiodors (III 7) der Überlieferung der drei andern sehr viel näher ge-

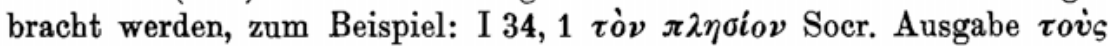
$\pi \lambda \eta \sigma i o v\left(\pi \varepsilon ́ \lambda \alpha_{\varsigma}\right.$ Sozom.) die übr., aber der Plural proximos Cass.; ebd. 2 


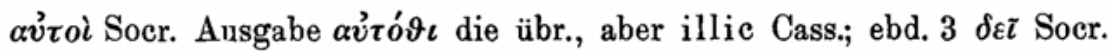

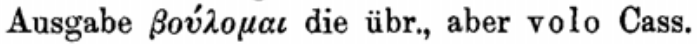

Es ist wohl nöglich, daß Sozomenus, abgesehen davon daß er Socrates las, wie gewöhnlich auf die Sammlung zurückgegriffen habe,

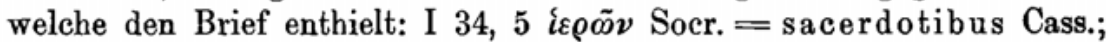

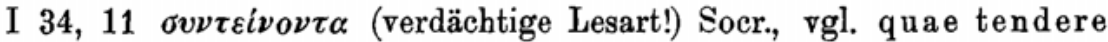
videntur Cass. - An diesen beiden Stellen hat Sozomenus wie Atha-

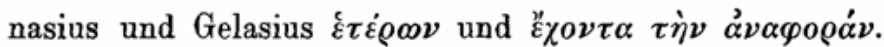

Diese letzteren Lesarten des Gelasius machen es unwahrscheinlich, wenigstens nach dem jetzt bekannten Texte des Socrates, daß er aus diesem schöpfe. Auch kann man nicht sagen, daß er von Sozomenus abhängig ist, da er ihn niemals copiert. Endlich stammt sein Text ebensowenig aus der Apol. c. Arian. des Athanasius. Ich zeige sogleich, daß Gelasius dieses Werk sicher nicht gekannt hat. Es bleibt also nur übrig, daß er den Brief direct aus einer Sammlung geschöpft hat. Wir wissen, $\mathrm{da} ß$ er eine solche Sammlung kannte, aus der z. B. die Briefe II 33 (vgl. Theodoret I 9) und ed. Commelin S. 59, $21 \mathrm{ff}$ (vgl. Theodoret I 20) entnommen sind.

Es bleibt jetzt noch zuzusehen, ob die Gelasius eigentümlichen Stellen auf den Originaltext zurückgehen oder ob sie willkürliche Zusätze sind. Ich glaube, man muß die zweite Erklärung annehmen.

Ganz allein die Version des Gelasius läßt Constantin voller Lobeserhebungen und Bewunderung für Athanasius erscheinen. Die Worte:

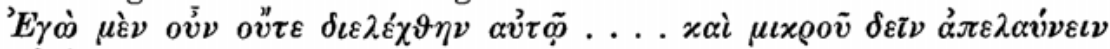

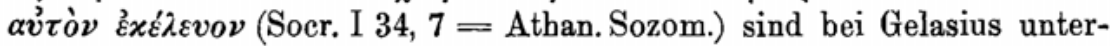
drückt, und zum Ersatz bietet er allein die folgenden Zeilen:

sSo gedemütigt und niedergeschlagen sahen wir den Mann, daß wir unaussprechliches Mitleid mit ihm empfanden, als wir erkannten, daß es Athanasius war, dessen heiliger Anblick imstande ist, selbst die Heiden zur Verehrung des Gottes des Weltalls zu gewinnen, er, den ehemals boshafte Menschen, Feinde des Friedens und der Eintracht, mit ungewöhnlichen falschen Anklagen umgarnten, so daß sogar ich selbst, durch ihren kunstvollen Trug mitfortgerissen, im Begriff war, mich gegen den Mann zu vergehen, wenn ich ihm nicht, durch göttliche Bestimmung getrieben, damals befohlen hätte, eilig aus Alexandrien an

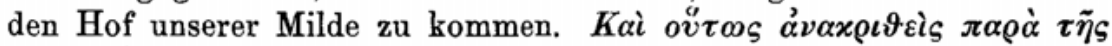

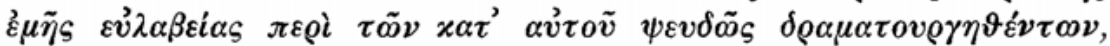

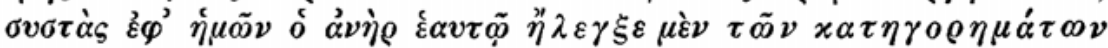

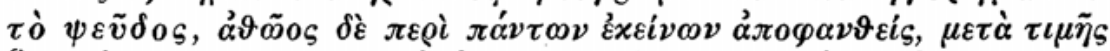

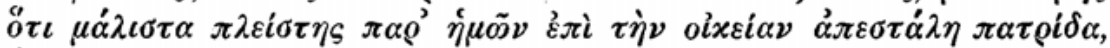

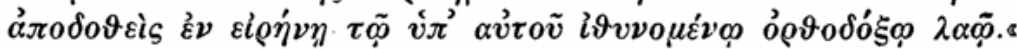


Es fehlt jeder Erklärungsgrund, warum Athanasius eine für ihn so schmeichelhafte Stelle sollte bescheiden unterdrückt haben. Die Einschiebung ist veranlaßt durch einen dem Gelasius eigenen Hang, seine Urkunden umzuschreiben, und durch seinen Wunsch, Constantin eine Lobrede auf Athanasius in den Mund zu legen.

Obendrein hat sich Gelasius noch, wie mir scheint, um diese ganze Erweiterung anzufertigen, einfach der Capitel Theodorets I 26 und 27 bedient, die er gerade eben abgeschrieben hatte (III 15 u. 16);

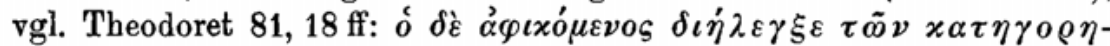

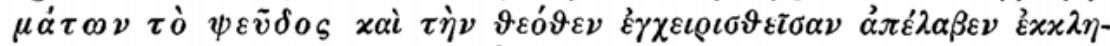
$\sigma i \alpha \nu$. Was bei Gelasius folgt: $\alpha \dot{\theta} \tilde{\omega} o \varsigma$ $\delta \dot{\varepsilon}$ usw. ist durch den Brief Constantins eingegeben, der von Theodoret unmittelbar hinter I 27 und von Gelasius selber III 16 wiedergegeben wird.

Auch Loeschcke würde wohl heute den Ähnlichkeiten keinen großen Wert mehr beilegen, die er in dem Briefe mit der Sprache Con-

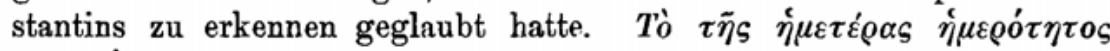

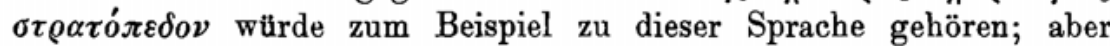
Gelasius hatte denselben Ausdruck anderwärts in dem Briefe (Socr. I $34,8)$ gefunden, wo er dafür Sorge getragen hatte, ibn umzugestalten

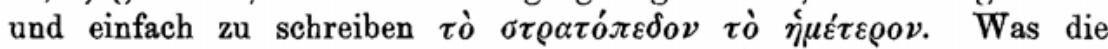

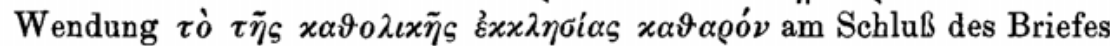
anlangt, so konnte auch sie wieder leicht unter dem Einfluß von $\tau \grave{o}$

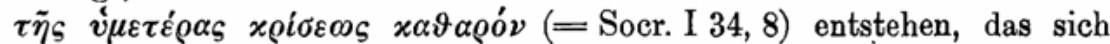
weiter oben findet.

Noch ein andres Beispiel möge hier eine Stelle finden. Es wird, glaube ich, nicht den geringsten Zweifel über den Grad des Vertrauens bestehen lassen, das wir zu den von Gelasius überlieferten Urkunden haben dürfen.

Gelasius III 15 (Ende) und 16 schreibt Theodoret I 26 von $\delta \delta \varepsilon$ ásıxó $\mu \varepsilon v o s$ an (S. 81, 18) ab und dann I 27 den Schluß von Constantins Brief an die Gemeinde von Alexandrien. Aber bevor Gelasius die letztere Anführung copiert, verläßt er einen Augenblick seine Quelle, um aus eigner Erfindung sich etwa in dem Sinne vernehmen $z u$ lassen: ¿Möge mir keiner der Leser vorwerfen, daß ich den Brief nicht ganz gebe. Ich habe vergeblich alle durchforscht, die über diese Ereignisse geschrieben haben, ich habe ihn nicht vollständig wiedergegeben gefunden ... Ich bin darüber noch trostloser als ihr alle. So also lautete der Schluß des Briefes."

Gelasius hat sehr schlecht gesucht. Der Brief findet sich vollständig bei Athanasius, Apologia contra A rianos 62, in einem Werk, das er sonach nicht durchforscht hat. 
Unter den Kirchenhistorikern enthalten weder Socrates noch Sozomenus jenen Schluß des Briefes. Dasselbe Bruchstück findet sich nur bei Theodoret I 27. Unzweifelhaft hat also Gelasius von diesem abgeschrieben.

Die Texte des Theodoret und Athanasius decken sich fast genau. Man betrachte, was Gelasius daraus gemacht hat. Ich übergehe einige willkürliche Überarbeitungen im Anfang, lege aber zur Vergleichung die folgenden Stellen vor:

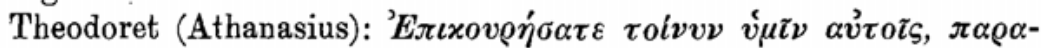

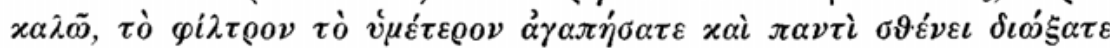

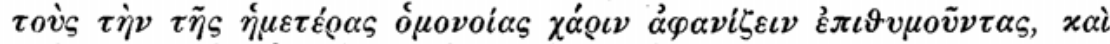

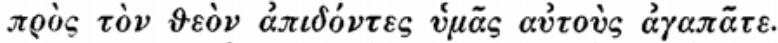

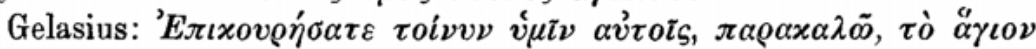

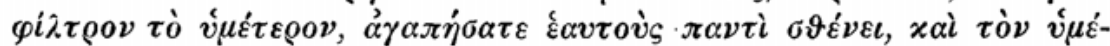

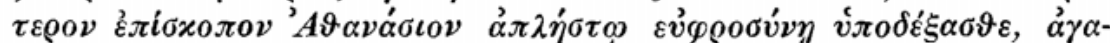

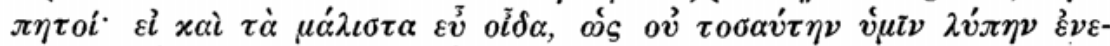

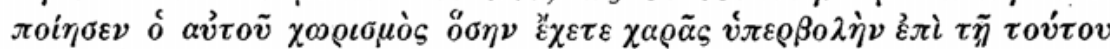

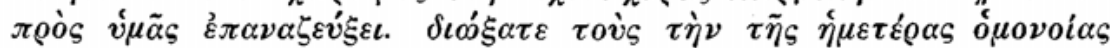

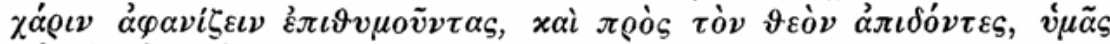
$\alpha \dot{v} \tau o \dot{v} \varsigma \dot{\alpha} \gamma \alpha \pi \dot{\eta} \sigma \alpha \tau \varepsilon, \pi \alpha \rho \alpha x \alpha \lambda \tilde{\omega}$.

Theodoret hat sieben oder acht Zeilen wiederzugeben unterlassen, die sich noch am Schluß des Briefes bei Athanasius finden. Gelasius fügt, weil er den Brief für abgeschlossen hält (wegen $\tau \grave{o} \tau \varepsilon \lambda \varepsilon v$ -

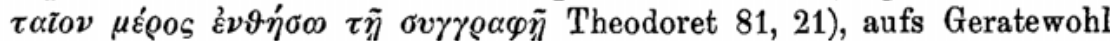
am Schluß seiner Copie die übliche Formel: $\delta$

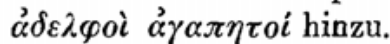

Eine andre Überarbeitung von Gelasius III 5 (Anleihe aus Theodoret I 17) habe ich in der Revue archéologique 1909, S. 50 nachgewiesen. Bei den Fälschungen der Urkunden scheint unter andern ein Zweck des Gelasius zu sein, die Meinung zu erwecken, daß er aus wertvollen, vor ihm unbekannten Quellen schöpfe.

In den Erzählungen gefällt sich Gelasius zuweilen ebenfalls darin, Lügen eigener Erfindung einzuführen. So erzählt er III 16 die Affäre der Synode von Antiochien und die Umtriebe gegen Eustathius ausschließlich nach Theodoret I 21. Dennoch behauptet er, daß er ältere Quellen besitze und die Ungenauigkeit seines Vorgängers verbessern werde, all das aus dem lächerlichen Interesse, zu beweisen, daß Eusebius von Caesarea in der Angelegenheit von Antiochien keine Rolle gespielt habe.

Diese wenigen Hinweise gentugen, um die Vorsicht zu begränden, die bei der Benutzung des Gelasius für den Aufbau von Theodorets Text anzuwenden ist. 